\title{
Nanodrugs used in cancer therapy
}

\author{
Katerina Kopeckovaa, Tomas Eckschlager ${ }^{b}$, Jakub Sirc ${ }^{c}$, Radka Hobzovac, Johana PIch ${ }^{b}$, Jan Hrabetab ${ }^{b}$ Jiri Michalek ${ }^{c}$
}

Cancer despite the introduction of new targeted therapy remains for many patients a fatal disease. Nanotechnology in cancer medicine has emerged as a promising approach to defeat cancer. Targeted delivery of anti-cancer drugs by different nanosystems promises enhanced drug efficacy, selectivity, better safety profile and reduced systemic toxicity. The article presents an overview of recent developments in cancer nanomedicine. We focus on approved anti-cancer medical products and on the results of clinical studies, highlighting that liposomal and micellar cytostatics or albuminbased nanoparticles have less side effects and are more efficient than "free" drugs. In addition, we discuss results of in vitro and in vivo preclinical studies with lipid, inorganic and polymer nanosystems loaded by anticancer drugs which according to our meaning are important for development of new nanodrugs. Pharmacokinetic characteristics of nanodrugs are discussed and characterization of major nanotechnology systems used for cancer nanomedicine is presented.

Key words: cancer, nanomedicine, nanotechnology, nanodrugs, targeted therapy

Received: July 6, 2018; Accepted with revision: March 25, 2019; Available online: April 9, 2019 https://doi.org/10.5507/bp.2019.010

${ }^{a}$ Department of Oncology, $2^{\text {nd }}$ Faculty of Medicine, Charles University and University Hospital Motol, Prague, Czech Republic ${ }^{b}$ Department of Pediatric Hematology and Oncology, $2^{\text {nd }}$ Faculty of Medicine, Charles University and University Hospital Motol, Prague, Czech Republic

'Institute of Macromolecular Chemistry, Academy of Sciences of Czech Republic

Corresponding author: Katerina Kopeckova, e-mail: katerina.kopeckova@fnmotol.cz

\section{INTRODUCTION}

Cancer treatment has undergone major advances in the last 30 years mainly due to the improved understanding of the process of carcinogenesis, cancer cell biology and the tumour micro-environment ${ }^{1}$. The introduction of targeted treatment based on small molecules or monoclonal antibodies has improved the prognosis of patients suffering from cancer. Despite the intense efforts in preclinical and clinical research, many advanced malignant tumours still remain fatal. One way for improving survival of cancer patients is therapy using nanocarriers for anticancer drugs. Nanoscience is defined by Yang et al. as a discipline that studies the phenomena and manipulation of materials at atomic, molecular and macromolecular level, where the properties differ significantly from those on a larger scale. Nanotechnologies are the design, characterization, production, and application of structures, devices and systems by controlling shape and size on the nanometer scale ${ }^{2}$.

One of the main limitations of systemic chemotherapy is low concentration of the drug in the tumour, its rapid clearance from the circulation and serious toxic effects outside the tumour ${ }^{3}$. Nanoparticles designed for tumour targeted therapies usually consist of nanocarrier and an active agent - drug, although nanoparticle formulations of the drug by itself are also possible ${ }^{4}$. The composition of the nanocarriers differs in terms of the used material like phospholipids, lipids, dextran, chitosan, or various synthetic polymers, carbon, silica, or metals. The main goals in the development of nanodrugs are both nonspecific (enhanced permeability and retention effect, see below) and specific targeting and delivery, better safety and bio- compatibility, and improved pharmacokinetic characteristics $^{4}$. Nanotechnology entered cancer treatment some decades ago. Several medical products were approved for clinical use such as albumin-bound-paclitaxel, liposomal doxorubicin and liposomal irinotecan (for more examples, see Tab 1). In addition, many anti-cancer nanodrugs are the subject of various phases of clinical trials and preclinical research ${ }^{5}$. However, the increased cost of nanodrugs, compared with free drugs is their main disadvantage ${ }^{5}$.

The aim of this literature overview, the results of our preclinical experiments and our clinical experiences with nanodrugs, is to present an overview of recent developments in cancer nanomedicine, to discuss pharmacokinetic characteristics of nanodrugs and to characterize major nanotechnology systems used for cancer nanomedicine.

\section{NANOMATERIALS FOR NANODRUGS}

Nanomaterials are characteristic by small sizes (1- $100 \mathrm{~nm}$ ), large ratio of surface area to volume, which may be orders of magnitude greater than that of macroscopic materials. Ideal nanomaterials for drug delivery should be non-toxic, biocompatible, blood stable, non-immunogenic and non-thrombogenic, and eventually biodegradable. Tumour-targeted nanomedicines (nanodrugs) are drug delivery systems developed to improve anti-cancer effects and particularly to overcome the toxicity of standard systemic chemotherapy ${ }^{5}$.

The history of nanoparticles began in the $1950 \mathrm{~s}$, when Jatzkewitz designed a polymer-drug conjugate ${ }^{6}$ followed by Bangham who discovered liposomes in the mid-1960s. In 1972, Scheffel and colleagues reported albumin based 


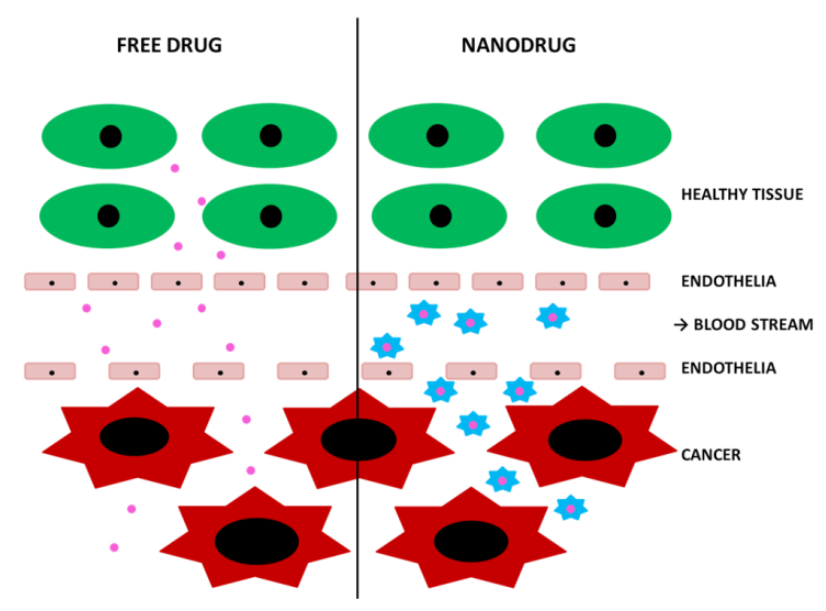

Fig. 1. The principles of EPR effect. Adapted from ${ }^{74,75}$.

nanoparticles that served as the basis of albumin-bound paclitaxel (Abraxane ${ }^{\circledR}$ ), the first nanodrug approved for clinical use ${ }^{7}$.

The systemic delivery of anti-cancer drugs is associated with toxicity to healthy tissue in the body. Nanotransporters containing anti-cancer drugs improve their therapeutic index, modify the pharmacokinetics and tissue distribution to increase drug delivery to the site of action and/or reduce the concentration in healthy tissues ${ }^{4}$. The administration of anti-cancer drugs directly to the tumour site can overcome side effects causing healthy tissues damage and increase the efficacy of the treatment by delivering higher doses of active drugs to the tumour site. Several systems which can be used as reservoirs of anti-cancer drugs for local chemotherapy were tested ${ }^{8}$. GLIADEL ${ }^{\circledR}$ wafer, approved by FDA and in several EU countries, is a biodegradable polymer (polifeprosan 20) implant containing the cytostatic drug carmustine with release rate over a 2 to 3 weeks. It is placed in the resected tumour bed of high grade glial tumours ${ }^{9}$.

\section{PHARMACOKINETIC CHARACTERISTICS OF NANODRUGS}

The nanodrug systems can offer several advantages like higher metabolic stability, higher membrane permeability, improved bioavailability and prolonged activity. Nanotransporters allow targeted delivery of the anti-cancer agents to the tissue as well as at the cellular level. For example, mucosal or transdermal absorption depends on size, surface charge and hydrophobicity. The size of the particles is a key factor; smaller nanodrugs (particles) are characterized by higher transcellular uptake than larger particles ${ }^{10}$. Nanoparticles larger than $300 \mathrm{~nm}$ cannot be absorbed by intestinal cells. Only nanoparticles smaller than $500 \mathrm{~nm}$ can penetrate the bloodstream ${ }^{11}$.

Most of the nanodrugs in the clinical practice use the concept of passive targeting. This mechanism refers to substantial extravasation of the nanodrugs into the interstitial fluid at the tumour site. The drugs are retained for a prolonged time at the tumour site due to aberrant

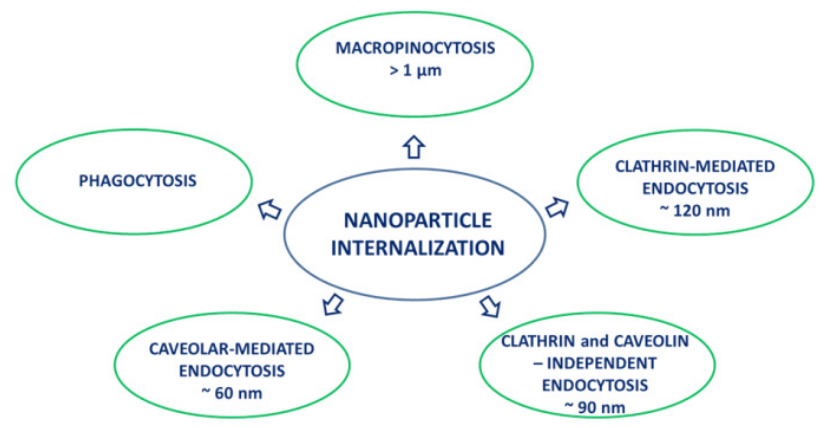

Fig. 2. Internalization of nanotransporters in cell. Adapted from $^{73,74}$.

blood and lymphatic vessel system in tumour micro-environment. This mechanism is referred to as "enhanced permeability and retention" (EPR) effect ${ }^{12}$ (Fig 1). The nanoparticles enter the tumour cells via diverse endocytotic pathways ${ }^{13}$ (Fig 2). The endocytosis-limited uptake appears to be the reason for overcoming the P-glycoprotein cell surface membrane efflux pump, responsible for the multidrug resistance phenotype ${ }^{14}$. The nanodrug is characterized by the release of the drug at the site of the tumour or directly in the cancer cell. Drugs are released by different mechanisms (diffusion, erosion, or degradation) which depends on the type of nanoparticles ${ }^{4,7}$.

The potency of the EPR effect depends on the size of the targeted nanodrug, tumour size and type. The nanodrug is active through EPR in the case of molecular weight $40-800 \mathrm{kDa}$ and size $20-100 \mathrm{~nm}^{15}$. Clinical studies measuring the accumulation of labelled liposomes in tumours have illustrated some concerns about diseasedependent access and/or accumulation of the nanodrug which can differ from tumour to tumour ${ }^{5}$. Recently tumour-associated macrophages have been proposed as a reservoir of nanoparticles from which the drug is gradually released to surrounding cells. However, some clinical trials have not confirmed the efficacy of the EPR effect because of insufficient vasculature and changes of extracellular matrix components ${ }^{16}$. Various biomarkers for EPR which would allow the prediction of nanodrug efficacy are under investigation. The ratio of matrix metalloproteinase 9 to tissue inhibitor of metalloproteinase 1 or vessel wall content were tested as a predictor of effective EPR (ref. ${ }^{17}$ ).

In active targeting, the anti-cancer agents are attached to ligands and bind specifically to structures-receptors on the target tumour cell (see Tab 2). A number of molecules e.g. transferrin-receptors (TfR), epidermal growth factor receptors (EGFR), folate receptors (FR), CD44 or CD22 have been tested for active targeting of nanodrugs ${ }^{18}$. For example, antibody targeted drug ado-trastuzumab emtansine (Kadcyla ${ }^{\circledR}$ ) is approved for the treatment of advanced HER2 positive breast cancer. The anti-tumour activity of these drugs depends on the expression and distribution of binding receptors, preferentially on tumour cells, the internalization of the conjugate mainly 
Table 1. Overview of approved anti-cancer nanodrugs. Adapted from ${ }^{8,72}$.

\begin{tabular}{lll}
\hline Name & Formula & Approved indication(s) \\
\hline DaunoXome & Liposomal daunorubicin & HIV-related Kaposi sa \\
Caelyx, Doxil & Pegylated liposomal doxorubicin & Breast, Ovarian ca, Kaposi sa, Multiple myeloma \\
DepoCyte & Liposomal cytarabine & Lymphomatous meningosis \\
Oncaspar & PEG asparaginase & Acute lymphoblastic leukemia \\
Abraxane & Albumin-bound paclitaxel & Breast, Pancreas ca, NSCLC \\
Myocet & Liposomal doxorubicin & Breast, Ovarian ca, Kaposi sa, Multiple myeloma \\
Marqibo & Liposomal vincristine & Acute lymphoblastic leukemia \\
Genexol & Paclitaxel loaded polymeric micelle & Breast, Ovarian ca, NSCLC \\
Onivyde & Liposomal irinotecan & Pancreas ca \\
Kadcyla & Trastuzumab linked to emtansine & HER2+ breast ca \\
Mepact & Liposomal mifamurtide & Osteosarcoma \\
Gliadel Wafer & Carmustine in poliferosan 20 & High grade glial tumours- local therapy \\
\hline
\end{tabular}

ca - carcinoma, sa - sarcoma

Table 2. Examples of targeting ligands. Adapted from ${ }^{73,74}$.

\begin{tabular}{llll}
\hline Type of ligand & Ligand & Receptor & Cancer \\
\hline Antibodies & Trastuzumab & Her2/neu & Breast, gastric, lung ca \\
& Rituximab & CD20 & B-cell NHL and leukemia \\
& Anti-CD19 & CD19 & B-cell NHL and leukemia \\
Aptamers & Pegaptanib & VEGF receptor & Different cancers \\
& A10 aptamer & PSMA & Prostate ca \\
& RGD & Integrin receptors & Different cancers \\
Peptides & ATWLPR & VEGF receptor & Different cancers \\
& Vasoactive intestinal peptide & VAP receptor & Different cancers \\
& Lyp-1 & P32 receptors & Different cancers \\
Proteins & Transferrin, Ferritin & Transferrin receptor & Different cancers \\
& LHRH & LHRH receptor & Breast, ovarian, prostate ca \\
& Folic acid & Folate receptor & Different cancers \\
Small molecules & Galactose & Asialoglycoprotein receptor & Hepatocellular ca \\
& Biotin & Biotin receptor & Different cancers \\
& Mannose & MRC1 mannose receptor & Different cancers \\
\hline
\end{tabular}

Table 3. Overview of nanoparticles platform for drug delivery systems. Adapted from ${ }^{57,74}$.

\begin{tabular}{|c|c|c|c|}
\hline Composition & Particle type & Size $(\mathrm{nm})$ & Properties \\
\hline Polymer & & $10-1000$ & Biodegradable \\
\hline Poly(amidoamine) & Dendrimer & $1-100$ & Biocompatible \\
\hline Lipid & Liposomes, micelles & $15-1000$ & $\begin{array}{l}\text { Biocompatible, carry hydrophobic drugs, } \\
\text { biodegradable }\end{array}$ \\
\hline Gold & Spheres, rods, shells & $10-100$ & Biocompatible \\
\hline Silica & Spheres, rods, mesoporous & $10-100$ & Biocompatible \\
\hline Carbon & Nanotubes, buckyballs, graphene, nanodiamonds & * & Biocompatible \\
\hline
\end{tabular}

* Carbon nanotubes- diameter 10-100 nm, length < $100 \mu \mathrm{m}$, nanodiamnonds - 5 nm.

via endocytosis and the absence of shedding antigens and receptors in the circulation. Other factors contributing to the activity of nanodrugs are affinity, molecular weight, valence and biocompatibility. When applied intravenously the surface of nanoparticles is rapidly covered by various proteins forming a so called "corona" 19 .

Nanodrugs have to avoid clearance through uptake by the reticuloendothelial system (RES) which occurs mainly for particles larger than $100 \mathrm{~nm}$. Uptake of nanodrugs by
RES is one of the obstacles for their use. On the other hand, accumulation of nanodrugs in tumour associated macrophages may increase the concentration of the drug at the site of tumour ${ }^{20}$. Coating of nanoparticles with hydrophilic and/or amphiphilic polymers such as polyethylene glycol (PEG) or with copolymers of polyethylene oxide and polypropylene oxide (i.e. poloxamines) or polysorbate 80 can reduce the uptake via macrophages ${ }^{21}$. Another approach is to coat nanoparticles with a mem- 
brane of erythrocytes, leukocytes or thrombocytes, thus camouflaging them from detection by the mononuclearphagocyte system ${ }^{22}$.

\section{NANODRUG RELATED TOXICITY}

One of the main goals in nanomedicine is to reduce the toxicity found in conventional systemic chemotherapy. However, this hurdle is not overcome even by approved nanodrugs. In some cases, nanoparticles tend to produce reactive oxygen species (ROS) and free radicals, resulting in oxidative stress, inflammation, DNA damage, formation of multinuclear cells, and fibrosis ${ }^{11}$. The toxicity is multifactorial and depends on the size and shape of the nanodrugs and on their physicochemical characteristics, surface properties, constituent leaching, and triggering the immune reaction ${ }^{23}$. The surface of the nanodrug tends to influence the toxicity rather than the absolute dose of the active substance ${ }^{24}$. Some cases of asthma, bronchitis, Alzheimer's disease, Parkinson disease and vascular events due to blood clotting have been described in the literature as adverse events of nanosystems ${ }^{25}$. Further safety studies are needed to address the issue of nanodrug acute and late toxicity.

\section{TYPES OF NANOTRANSPORTERS}

The development of a broad range of nano-sized delivery systems with the ability to size, composition and functionality has provided a significant resource for nanomedicine. Overview of core materials and matrices is shown in Table 3.

\section{Lipid nanosystems}

Emulsions, liposomes and solid lipid-based nanoparticles belong to lipid nanosystems, and some of these medical products have been approved and have become a part of clinical practice. In general, the lipid- based carriers are well tolerated by humans since they are composed of physiological constituents ${ }^{26}$. On the other hand, some concerns regarding toxicity persist with the use of particular emulsifiers ${ }^{27}$.

Self-emulsifying drug delivery systems (SEDDSs) consist of mixtures of oil, surfactant, co-solvent, and solubilized drug. Their advantage is improved oral bioavailability of poorly water-soluble drugs, mainly for highly lipophilic drugs ${ }^{28}$. On the other hand, a potential disadvantage of emulsion systems is the rapid increase of systemic exposure resulting in toxicity due to the fast gastric emptying of SEDDSs. This can be overcome using a sustained drug release system ${ }^{29}$. Examples of these systems in clinical use are two SEDDS formulations of cyclosporine A ( CsA) developed by Novartis, Sandimmune ${ }^{\circledR}$ and Neoral ${ }^{\circledR}$. Besides an oral formulation, an inhalation emulsion formulation of CsA has been developed and tested for asthma therapy using animal models. Pharmacokinetic studies demonstrated that systemic exposure of CsA after intratracheal administration at an effective dose was fifty times lower than after systemic administration ${ }^{30}$. One may speculate that inhalation of cytostatics or targeted drugs in emulsion could be effective in lung tumours and/or metastases.

Liposomes are aqueous microcapsules surrounded by multilayer structures consisting of phospholipids or cholesterol. They are classified into small unilamellar vesicles ( 25 to $50 \mathrm{~nm}$ in diameter), large unilamellar vesicles and multilamellar vesicles (several lipid layers separated one from another by a layer of aqueous solution). The diameter size of large and multilamellar vesicles is about 100-150 nm. The aqueous compartment can load hydrophilic agents and the lipid part of hydrophobic agents. The composition of liposomes resembling the cell membrane makes them biocompatible. Non-specific uptake within a few minutes to few hours by RES, rapid clearance and opsonisation are obstacles to be overcome in clinical development of liposomal drugs. The pharmacokinetic characteristics of liposomes depends on their size, surface charge, membrane lipid packing, steric stabilization, dose and route of administration. In liposomal drugs the clearance and volume of distribution $\left(\mathrm{V}_{\mathrm{D}}\right)$ decreases, while the terminal half-life $\left(\mathrm{t}_{1 / 2}\right)$ and area under the plasma concentration curve (AUC) increases ${ }^{31}$.

Various functional ligands may be used to modify the characteristics of the liposome surface. PEG modification of the liposomal layer changes the size and charge of the systems which improves the drug delivery task. These delivery nanosystems protect the loaded drugs from degradation and prevent undesirable exposure to the drug environment and delay active agent release. Liposomes protect loaded drug from degradation by plasma proteins and reduce the drug leakage. PEG modification of liposomes increased systemic $t_{1 / 2}$ for the encapsulated drug caused by reduced uptake in the RES (ref. ${ }^{31}$ ). The PEGmodified liposomes also increase the efficacy through the EPR effect. The liposome systems are very attractive for drug development due to their specific biopharmaceutical properties such as high encapsulation efficiency for hydrophilic and hydrophobic agents, protection of encapsulated drugs from undesirable effects of surrounding environment, conjugation with specific active ligands for targeting therapy, prolonged systemic circulation, and modification of size and surface charge $\mathrm{c}^{32,33}$.

Examples of approved liposomal drugs used in oncology are amphotericin B - Abelcet ${ }^{\circledR}$ (antimycotic drug frequently used in mycotic infections during chemotherapy, liposomal amphotericin is less nephrotoxic than free drug which may be significant in cancer patients who experience kidney damage due to chemotherapy and also suffer from systemic mycosis during chemotherapy), doxorubicin - Myocet ${ }^{\circledR}$ and pegylated liposomal doxorubicin- Doxil ${ }^{\circledR}$ and Caelyx ${ }^{\circledR}$, cytarabine - DepoCyte ${ }^{\circledR}$, or irinotecan - Onivyde ${ }^{\circledR}$.

Doxil $^{\circledR}$ is characterized by prolonged circulation time and avoidance of RES uptake. The AUC is 300-fold greater than that of free drug. The clearance is reduced to at least 250 times and $V_{D} 60$ times lower. These changes of the pharmacokinetics translate into a better cardiotoxicity profile $^{34}$. A phase III study comparing liposomal and free 
Table 4. The pharmacokinetic parameters of total irinotecan and SN-38 (active metabolite of irinotecan) in humans. Adapted from ${ }^{40}$.

\begin{tabular}{|c|c|c|c|}
\hline \multirow[t]{2}{*}{ Analyte } & Parameter & ONIVYDE & Irinotecan \\
\hline & [unit] & $80 \mathrm{mg} / \mathrm{m}^{2}$ & $125 \mathrm{mg} / \mathrm{m}^{2}$ \\
\hline \multirow[t]{5}{*}{ Irinotecan } & AUC [h ng/mL] & $9.1 \times 10^{5}$ & $1.1 \times 10^{4}$ \\
\hline & $\mathrm{C}_{\max }[\mathrm{ng} / \mathrm{mL}]$ & $2.8 \times 10^{4}$ & $1.5 \times 10^{3}$ \\
\hline & Clearence $\left[\mathrm{L} / \mathrm{h} / \mathrm{m}^{2}\right]$ & 0.009 & 13.0 \\
\hline & $\mathrm{V}_{\mathrm{D}}\left[\mathrm{L} / \mathrm{m}^{2}\right]$ & 2.6 & 138 \\
\hline & $\mathrm{t}_{1 / 2 \text { effective }}[\mathrm{h}]$ & 20,8 & 6.1 \\
\hline \multirow[t]{3}{*}{ SN-38 } & AUC [h ng/mL] & 341 & 267 \\
\hline & $\mathrm{C}_{\max }[\mathrm{ng} / \mathrm{mL}]$ & 3.0 & 27.8 \\
\hline & $\mathrm{t}_{1 / 2 \text { effective }}[\mathrm{h}]$ & 40.9 & 11.7 \\
\hline
\end{tabular}

$\mathrm{AUC}=$ area under the plasma concentration curve (extrapolated to infinity for ONIVYDE and AUC 24h for non-liposomal irinotecan), $\mathrm{C}_{\max }=$ maximum plasma concentration, $\mathrm{t}_{1 / 2 \text { effective }}=$ effective half-lives, $\mathrm{V}_{\mathrm{D}}=$ volume of distribution

doxorubicin in patients with breast cancer found lower risk of cardiomyopathy, reduced grade 4 neutropenia and comparable anti-tumour activity even in women with previous anthracycline therapy ${ }^{34,35}$. Further decrease of cardiotoxicity, myelotoxicity and vomiting was achieved by pegylation of liposomal doxorubicin- Doxil ${ }^{\circledR}$, Caelyx $^{\circledR 36,37}$. The Jerusalem study which compared Doxil ${ }^{\circledR}$ to free doxorubicin showed much higher levels of doxorubicin both in tumour cells and tumour interstitial fluids after Doxil $^{\circledR}$ administration compared to free doxorubicin ${ }^{34}$. A pharmacokinetic study showed that the plasma elimination time of Doxil ${ }^{\circledR}$ followed a bi-exponential curve, with median values of half-lives of 2 and $45 \mathrm{~h}$, most of the dose being cleared from plasma under the longer half-life. A large difference in $\mathrm{V}_{\mathrm{D}}$ was also found (4 $\mathrm{L}$ for Doxil ${ }^{\circledR}$ versus $254 \mathrm{~L}$ for free doxorubicin). Similarly, doxorubicin derived from Doxil ${ }^{\circledR}$ showed a much slower clearance $\left(0.1 \mathrm{~L} / \mathrm{h}\right.$ for Doxil ${ }^{\circledR}$ vs. $45 \mathrm{~L} / \mathrm{h}$ for free doxorubicin) $\left(\right.$ ref. $\left.^{34}\right)$. Overall, there is strong evidence that liposomal doxorubicin is associated with a reduced risk of cardiomyopathy ${ }^{34-37}$. The recommended cumulative dose of Doxil ${ }^{\circledR}$ is $860 \mathrm{mg} / \mathrm{m}^{2}$ while of free doxorubicin it is 550 $\mathrm{mg} / \mathrm{m}^{2}$ and after mediastinum irradiation only $440 \mathrm{mg} /$ $\mathrm{m}^{2}$ (Recommendation Comprehensive Cancer Center, General Teaching Hospital \& First Faculty of Medicine, Charles University, Prague). Exceeding the cumulative dose of anthracycline significantly increases the risk of developing cardiomyopathy ${ }^{38}$.

In a clinical trial in lymphomatous meningitis comparing intrathecal administration of DepoCyte ${ }^{\circledR}$ (liposomal cytarabine) with standard cytarabine, $72 \%$ patients responded to DepoCyte ${ }^{\circledR}$ compared to $18 \%$ response rate to free drug. Moreover, DepoCyte ${ }^{\circledR}$ administration improved Karnofsky performance status scale at the end of induction treatment compared to free drug. The major side effects on both arms were headache and arachnoiditis, which were probably caused by the disease ${ }^{39}$. Liposomal irinotecan Onivyde ${ }^{\circledR}$ was approved for the treatment of advanced pretreated pancreatic cancer. The higher rate of accumulation at the tumour site and prolonged clearance are associated with the better survival of patients suffering from advanced or metastatic pancreatic cancer treated by
Onivyde ${ }^{\circledR}$ with 5-fluouracil compared to patients treated by monotherapy with 5-fluorouracil. The PEPCOL trial (study of Onivyde ${ }^{\circledR}$ or free irinotecan in combination with leucovorin and 5-fluorouracil in patients with metastatic colorectal cancer) showed encouraging tumour response and fewer side effects (namely diarrhoea and neutropenia) in the Onivyde ${ }^{\circledR}$ arm. Comparison of pharmacokinetic data of free and liposomal irinotecan is shown in Table 4 $\left(\right.$ ref. $\left.{ }^{40}\right)$.

Solid lipid nanoparticles are colloidal nanoparticles composed of triglycerides, diglycerides, monoglycerides, solid fats, or waxes stabilized by surfactant. They were developed as an alternative to liposomal formulations in order to improve physical stability, modulate release of the loaded drug, reduce their cost, and simplify manufacturing ${ }^{41}$. Unlike liposomes, they can be administered by various routes of application e.g. intravenously, orally, by inhalation, transdermally, nasally, intravesically etc. ${ }^{42}$ Current preclinical experiments show that solid lipid based anti-cancer drug systems seem to be superior to conventional drug solutions and are at least comparable to other encapsulated systems in many aspects such as drug efficacy, pharmacokinetics and drug biodistribution $^{43}$. However, clinical studies have yet to be conducted in this area.

\section{Inorganic nanomaterials}

A variety of inorganic nanosystems are under clinical development for radiotherapy and tumour diagnostics quantum dots, supermagnetic iron oxide, gold and hafnium oxide nanoparticles, and carbon nanotubes. The small size of particles (10-100 nm) enables penetration of capillaries and facilitates uptake in targeted tissue. Toxicity caused by membrane damage and induction of oxidative stress and instability were observed in the development of gold, silica and iron nanoparticles. Gold nanoparticles absorb light and convert photon energy into heat, which makes them suitable for hyperthermic therapy. Carbon nanotubes are carbon cylinders which can serve as carriers for drugs. Recently nanodiamonds (about $5 \mathrm{~nm}$ size) and graphene have been studied for cancer therapy in several preclinical studies ${ }^{44}$. Nanodiamonds can be loaded 
with cytostatics and functionalized. They carry drug to metastatic tumour cells as proven in animal experiments or they can be used as tracers that label cancer cells ${ }^{45}$. Chitosan modified single walled carbon nanotubes targeted by folic acid with doxorubicin were more effective and less toxic than free drugs in human hepatocellular cancer xenotransplantat in nude mice ${ }^{46}$.

Nanoparticles labelled with different isotopes have been investigated for tumour imaging using single-photon emission computed tomography, computed tomography and positron emission tomography ${ }^{47}$.

\section{Polymer nanosystems}

Polymers can be divided into natural polymers (i.e. proteins, peptides, glycans, starches, or cellulose) and synthetic ones. In the biomedical field, the latter are particularly represented by biocompatible or biodegradable polyesters such as poly(lactic acid) (PLA), poly(lacticco-glycolic acid) (PLGA), or polycaprolactone (PLC). Various forms of polymer nanosystems intended for cancer therapy are under preclinical investigation, including nanoparticles, nanosponges, dendrimers, micelles, nanogels or nanofibers ${ }^{48}$.

Polymeric nanodrugs provide an effective way for encapsulation of the drug while protecting the drug against degradation. Moreover, compared to other nanodrugs, they are characterized by higher stability, multiple available routes of administrations and possibility of adjusting the controlled drug release with prolonged drug action. Another beneficial feature is their biodegradability, low immunogenicity and low toxicity ${ }^{49}$. The active compounds may be released to the target origin by resorption, diffusion through the polymer matrix or by the matrix degradation.

\section{Nanoparticles}

Naturally occurring polymers are used for synthesis of nanoparticles. Albumin-based nanoparticles containing paclitaxel - Abraxane ${ }^{\circledR}$ - was approved for clinical practice for the treatment of breast, non-small lung (NSCLC) and pancreatic cancer. The pharmacokinetics data showed higher distribution volume, so greater extravascular distribution can be anticipated compared to free paclitaxel. Based on the better efficacy data, Abraxane ${ }^{\circledR}$ was approved for all three indications; as monotherapy in case of breast cancer or in combination with gemcitabine for pancreatic cancer and with carboplatin for NSCLC. A randomized trial in patients with metastatic pancreatic cancer which used either Abraxane ${ }^{\circledR}$ plus gemcitabine or gemcitabine alone demonstrated significant prolongation of overall and progression-free survival for patients receiving Abraxane ${ }^{\circledR}$. The incidence of adverse reactions, including neutropenia, fatigue, peripheral neuropathy, nausea, alopecia, peripheral edema, diarrhea, pyrexia, vomiting, decreased appetite, rash, and dehydration, was significantly higher in patients who received nanoparticles plus gemcitabine ${ }^{50}$. The clinical trial in patients with advanced NSCLC demonstrated higher overall response rate in patients randomized to the treatment with Abraxane in combination with carboplatin compared to the combination with free paclitaxel, however the difference in overall survival between these two groups was not significant ${ }^{49}$. Serious adverse reactions occurred equally in both groups. Another study was conducted in patients with metastatic breast cancer. Patients were randomized to either Abraxane ${ }^{\circledR}$ or free paclitaxel. Objective response rate was almost twice as high in the nanoparticle group than in the free paclitaxel group and the incidence of clinically important adverse events was similar in both groups $^{50}$.

Another protein-based polymer which can be used as drug nanotransporter is apoferritin (Apo), a naturally occurring iron-storage protein consisting of 24 protein subunits, which is responsible for the storage and transfer of iron, and can provide the much needed properties of a drug-nanocarrier. Apo binds to transferrin receptors and/or SCARA5 receptors that are overexpressed in several malignant tumours and thereafter is internalized. Apo as a nanocarrier has the potential to move undetected through the body without being recognized by the immune system of the patient. Furthermore, this natural protein can be modified with recognition ligands to achieve tumourspecific targeting. These extra modifications can increase the concentration of the drug in the tumour as detected in both in vitro and in vivo experiments with doxorubicin loaded Apo (ref. ${ }^{51,52}$ ).

\section{Nanosponges}

Nanosponges are a novel class of hyper-crosslinked polymer based colloidal structures consisting of solid nanoparticles with colloidal cavities in which drugs can be encapsulated. The spherical shape and a size of < $500 \mathrm{~nm}$ makes them ideal for preparing various application forms like topical, parenteral, aerosol and tablets ${ }^{53}$. $\beta$-cyclodextrin based nanosponges with paclitaxel were tested in vitro using oropharyngeal spinocellular cancer cells and seem to be promising. Paclitaxel-loaded nanosponges were safe. Increased amounts of paclitaxel entering cancer cells and enhanced anticancer effects of paclitaxel were observed ${ }^{54}$.

\section{Dendrimers}

Dendrimers are highly symmetric, spherical compounds composed of repetitively branched molecules ranging from 1-100 $\mathrm{nm}$. They differ from linear polymers by an architecture with tailor-made surface groups. Their properties are mainly determined by the functional groups on their surface. They can be used as a backbone for different biological material for targeted therapy and diagnostics. Their advantages are biocompatibility, easy elimination from the body and significantly expressed EPR effect. The drawback is the cytotoxicity to normal cells resulting from the physiological stability of cationic groups of dendrimers ${ }^{55}$. An experiment published by Lee et al. with doxorubicin conjugated to a biodegradable dendrimer demonstrated better anti-tumour effect than with the free doxorubicin and similar effect like an equimolar dose of liposomal form of doxorubicin (Doxil ${ }^{\circledR}$ ) in mice bearing colon carcinoma ${ }^{56}$. 


\section{Micelles}

Micelles are particles with the size of several tens of nanometers and with hydrophobic tail (PEG 2-15 $\mathrm{kDa}$ ) and hydrophilic head (poly-esters, polyethers, or polyamino acids) which are usually used as carriers of hydrophobic drugs and can be applied directly into the circulation like liposomes or via inhalation or transdermally. Pulmonary drug delivery offers the possibility of local targeting for the treatment of lung cancer and/or metastasis. Their advantage is relative higher molecular weight that enables preferred storage in the tumour tissue via EPR. Micelles can be an alternative to liposomes in terms of passive and active targeting. They can improve absorption and distribution of drugs and avoid opsonisation and phagocytic clearance by RES (ref. ${ }^{57}$ ). Different cytostatic drug-loaded micelles (doxorubicin, paclitaxel, curcumin) were successfully tested in vitro and in vivo (for overview see ${ }^{58}$ ). Moreover, paclitaxel encapsulated inside micelles was tested in a clinical phase I study in patients with advanced malignancies with tolerable toxicity. The safety profile was better than that of free paclitaxel ${ }^{59}$.

\section{Nanofibers}

Nanofibers are currently extensively studied in the medicinal field as their internal structure with relatively high surface-to-volume ratio and microporosity provides numerous opportunities to design drug delivery systems for various therapeutics including anti-cancer agents ${ }^{55}$. The appropriate composition of the polymer matrix allows the incorporation and subsequent release of various, hydrophilic as well as hydrophobic active agents. The most studied polymers for preparation of nanofibrous delivery systems are PLA, PLGA, or PCL. The release of the hydrophobic drugs with low solubility in aqueous environment can be further modified by addition of amphiphilic polymers such as PEG (ref. ${ }^{60}$ ). Several studies have been published on the incorporation of anti-cancer drugs such as doxorubicin ${ }^{61}$, paclitaxel ${ }^{62}$, camptothecin ${ }^{63}$, or cispla$\operatorname{tin}^{64}$. They have not yet been tested systemically but only for topical application, usually after surgery. Localized chemotherapy, delivered directly to the affected area, was found to be a promising approach for treatment of various malignant tumours such as glioma ${ }^{62}$, breast ${ }^{65}$, liver $^{63}$, cervical $^{64,65}$ or lung cancer ${ }^{64,66}$. Nanofibers have been successfully tested for topical adjuvant chemotherapy in the animal model. Testing of poly(D, L-lactide)/polyethylene glycol nanofibers loaded by paclitaxel both in vitro and in vivo has been conducted by our group. We found decreased recurrence after surgery in xenotransplants of human fibrosarcoma in mice models of recurrent fibrosarcoma after implantation of paclitaxel loaded PLA nanofibers compared to systemic paclitaxel administration (Hrabeta et al, unpublished results). Their morphology, transport properties, drug delivery curves under various conditions and tests in vitro and in vivo seem to be promising in local therapy ${ }^{67}$.

\section{THERANOSTIC}

Theranostic nanomedicine is the term used for the combination of diagnostic and therapeutic functions into one system. An example of theranostics is the study by Harrington et al. in which ${ }^{111}$ In-DTPA-labeled pegylated liposomes were studied in patients with advanced breast, head and neck, lung, cervical cancer and glioblastoma and whole body scintigraphy was used to detect the distribution of labeled liposomes. Images were positive in 15 of 17 patients ( 1 negative was in the case of lung and breast cancer). The authors assume, that pegylated liposomes seem to be promising for theranostic medicine for solid tumours ${ }^{47}$.

\section{ENHANCING THE ACTIVITY OF CANCER NANOMEDICINE}

Despite major advances in nanoscience and the introduction of some nanodrugs into clinical practice their real benefits have still yet to be realised. The main goal is to enhance drug delivery to the tumour site. Pharmacokinetic parameters can be affected by formation of corona after the nanosystems enter the circulation. We need to explore specific models mimicking in vivo processes in terms of serum protein interaction, tumour microenvironment and extracellular matrix. Enhancing extravasation of the nanoparticles from the systemic circulation should be translated in enhanced anti-tumour effect. The next crucial factor is penetration of the nanosystems into the tumour. This depends on the size and binding activity of the macromolecules and additionally effecting kinetics of the nanosystems. Internalization and intracellular trafficking of the nanosystems also play an important role in the antitumour effect. We believe that the further investigation of passive and active targeting is the most promising approach in the development of new anticancer nanodrugs. Targeting enhances drug delivery to the tumour site and improves penetration of the drugs into the cancer cells.

Controlled drug release is another important factor for effective cancer treatment. Several pharmacokinetic parameters should be studied in detail when designing nanosystems for anti-cancer drug such as maximum serum concentration achieved after administration $\left(\mathrm{C}_{\max }\right)$ and AUC, specifically the correlation between drug $\mathrm{C}_{\text {max }}$ and nanoparticles $\mathrm{C}_{\max }$, and/or between drug plasma AUC and nanoparticles plasma AUC. The major clinical implication unlike the free drug is the toxicity from the nanoparticle AUC (ref. ${ }^{68}$ ). To be successful in fighting cancer we have to take further steps towards more effective targeting the microenvironment of the primary tumour and metastases.

\section{CONCLUSION AND FUTURE DIRECTIONS}

Our review summarizes the role of nanoparticles for anticancer drug delivery as one of the most advanced aspects of nanomedicine. However, research is also ongoing 
into the use of nanoparticles in other therapeutic procedures such as immunotherapy or gene therapy. Intensive research is ongoing in nanomaterials for antisense nucleotide and for anti-cancer immunotherapy. Liposomal antisense oligonucleotides selectively inhibiting diseasecausing genes seems promising for cancer therapy.

Liposomal bcl-2 antisense oligonucleotide inhibited follicular lymphoma cells in vitro ${ }^{66}$. Degradation, inefficient cellular uptake and induction of immune reactions represent the main disadvantages of antisense nucleotides. The antisense nucleotides loaded nanoparticles seem to overcome these limitations ${ }^{65}$.

Some nanoparticles can act as an antigen reservoir for loading of dendritic cells. Antigen encapsulation into PLGA particles increases the efficiency of antigen presentation by dendritic cells because PLGA microspheres deliver antigen more efficiently than soluble antigens or antigens conjugated to non-degradable beads ${ }^{65}$. Moreover, nanoparticles may be used in oncology for hyperthermic therapy (magnetic nanoparticles heated by a magnetic field) or with radioactive isotopes for systemic radiotherapy of tumours ${ }^{65}$.

Tremendous advance has been achieved during the last two decades in cancer nanomedicine. Some medical products have been approved (see Table 1) and have been included in clinical practice. But the real revolution in cancer therapy using nanodrugs is still awaited. We need to understand more about the EPR, interactions of nanoparticles with cells, targeting tumour and the metastatic microenvironment. Better understanding of nanodrug biodistribution, pharmacokinetics, toxicity and their role in therapeutic protocols is warranted, in order for them to become part of standard treatment algorithms. Untoward immunological reactions also require careful consideration when using this technology. Not only nanodrugs containing classic cytostatics, but incorporation of small targeted molecules, siRNA, antisense oligonucleotides, and DNA inhibitor oligonucleotides can enhance the effectivity of nanomedicine.

Further, the combination with new drugs of immunotherapy creates an opportunity for nanosystems to improve anti-cancer immunity. We need to perform controlled, reproducible and scalable nanoparticle synthesis. Addressing these main tasks, we can expect new theranomedicine products for better treatment and tumour imaging.

Still some hurdles for nanodrugs have to be overcome. Most of these drug systems have undergone some in vitro and in vivo testing. However, we await the data from more clinical trials with nanodrugs. Only these results can confirm the efficacy and safety in clinical settings. Each of the nanodrug platform is distinctive and needs to be assessed experimentally and clinically as a new system. The stability of nanoparticles, size uniformity, a controlled drug release rate, preparations in a large scale according to good manufacturing practice and the manufacturing cost have to be addressed in order to make them available to clinical practice.

In conclusion the results of clinical studies demonstrate that liposomal forms of cytostatics or albumin- based nanoparticles are associated with fewer side effects and can be more efficient than "free" drugs. Moreover, for local therapy of brain tumours, GLIADEL ${ }^{\circledR}$ wafer biodegradable polymer implant containing the active carmustine was licensed. One may speculate that various other nanotransporters like inorganic nanoparticles, dendrimers, nanosponges, micelles or nanofibers will also decrease side effects and/or improve the efficacy of anti-cancer drugs and may improve the efficacy of immunotherapy and therapy by antisense nucleotides. There are exciting times ahead for this dynamic field which will result in benefits to patients with cancer.

\section{Search strategy and selection criteria}

Our research strategy was aimed at evaluating theoretical, preclinical and particularly clinical studies of anti-cancer nanodrugs. Scientific articles to April 2018 were searched for keywords nanodrugs or nanocarrier and cancer or tumor using databases PubMed and Web of science, only English language papers were reviewed.

Acknowledgment: This work was supported by the Grant Agency of the Czech Republic [grant number 1604863S]; the Ministry of Education, Youth and Sports of the Czech Republic within the National Sustainability Program II [project BIOCEV-FAR LQ1604, "BIOCEV" CZ.1.05/1.1.00/02.0109]; and by the Ministry of Heath of the Czech Republic for the conceptual development of research organization, University Hospital Motol, Prague, Czech Republic [grant number 00064203].

Author contributions: KK: incited the review, prepared and summarized the clinical related parts of the manuscript; TE: prepared and summarized the preclinical related parts of the manuscript, searched the literature; JH, JP: literature search for preclinical and clinical and prepared Fig. 1 and 2; JS, RH: literature search for chemical and technical parts of the manuscript; JM: prepared and summarized the chemical and technical parts of the manuscript.

Conflict of interest statement: The authors state that there are no conflicts of interest regarding the publication of this article.

\section{REFERENCES}

1. Hanahan D, Weinberg RA. Hallmarks of Cancer: the next generation. Cell 2011;144:646-74

2. Yang W, Peters Jl, Wiliams RO. Inhaled nanoparticles- a current review. Int J Pharm 2008;356:239-47.

3. Lammers T, Hennink WE, Storm G. Tumour-targeted nanomedicines: principles and practice. Br J Cancer 2008;99:392-7.

4. De Jong WH, Borm PJ. Drug delivery and nanoparticles: applications and hazards. Int J Nanomedicine 2008;3:133-49.

5. Hare Jl, Lammers T, Ashford MB, Puri S, Storm G, Barry ST. Challenges and strategies in anti-cancer nanomedicine development: An industry perspective. Adv Drug Deliv Rev 2017;108:25-38.

6. Jatzkewitz H. Ein Kolloidales Blutplasma-Ersatzmittel (Pollyvinylpyrrolidon) Gebundenes Peptamin (Glycyl-L-leucyl-Mezcalin) Als Neuartige Depotform Fur Biologisch Aktive Primare Amine (Mezcalin). Zeitschrift Fur Naturforschung Part B-Chemie Biochemie Biophysik Biologie Und Verwandten Gebiete 1955;10:27-31.

7. Aslan B, Ozpolat B, Sood AK, Lopez-Berestein G. Nanotechnology in cancer therapy. J Drug Target 2013;21:904-13. 
8. Krukiewicz K, Zak JK, Biomaterial-based regional chemotherapy: Local anticancer drug delivery to enhance chemotherapy and minimize its side-effects, Mater Sci Eng C 2016;62:927-42.

9. Perry J, Chambers A, Spithoff K, Laperriere N. Gliadel wafers in the treatment of malignant glioma: a systematic review. Curr Oncol 2007;14:189-94.

10. Roger E, Lagarce F, Garcion E, Benoit JP. Biopharmaceutical parameters to consider in order to alter the fate of nanocarriers after oral delivery. Nanomedicine 2010;5:287-306.

11. Ai J, Biazar E, Jafarpour M, Montazeri M, Majdi A, Aminifard S, Zafran M, Akbari HR, Rad HG. Nanotoxicology and nanoparticle safety in biomedical design. Int J Nanomedicine 2011;6:1117-27.

12. Duncan R. Polymer conjugates as anticancer nanomedicines. Nat Rev Cancer 2006;6:688-701.

13. Vega-Vila KR, Takemoto JK,Yánez JA, Remsberg CM, Forrest ML,Davies NM. Clinical toxicities of nanocarrier systems. Adv Drug Deliv Rev 2008;60:929-38.

14. Rihova B, Kubackova K. Clinical Implication of N-(2-hydroxypropyl) methacrylamide copolymers. Curr Pharm Biotechnol 2003;4:311-22.

15. Schmidt MM, Wittrup KD. A modeling analysis of the effects of molecular size and binding affinity on tumour targeting. Mol Cancer Ther 2009;8:2961-871.

16. Huynh E, Zheng G. Cancer nanomedicine: addressing the dark side of the enhanced permeability and retention effect. Nanomedicine 2015;10:1993-5.

17. Yokoi K, Tanei T, Godin B, van de Ven AL, Hanibuchi M, Matsunok A, Alexander J, Ferrari M.. Serum biomarkers for personalization of nanotherapeutics-based therapy in different tumor and organ microenviroments. Cancer Lett 2014;345:48-55

18. Piktel E, Niemirowicz K, Wątek M, Wollny T, Deptuła P, Bucki R. Recent insights in nanotechnology-based drugs and formulations designed for effective anti-cancer therapy. J Nanobiotechnology 2016;14:39.

19. Monopoli MP, Aberg C, Salvati A, Dawson KA. Biomolecular coronas provide the biological identity of nanosized materials. Nat Nanotechnol 2012;7:779-86.

20. Bolkestein $M$, de Blois E, Koelewijn SJ, Eggermont AM, Grosveld F, de Jong M, Koning GA. Investigation of Factors Determining the Enhanced Permeability and Retention Effect in Subcutaneous Xenografts. J Nucl Med 2016;57:601-7.

21. Jokerst JV, Lobovkina T, Zare RN, Gambhir SS. Nanoparticle PEGylation for imaging and therapy. Nanomedicine 2011;6:715-28.

22. Hu CM, Zhang L, Aryal S, Cheung C, Fang RH,Zhang L. Erytrocyte membrane-carmouflaged polymeric nanoparticles as a biomemetic delivery. Proc Natl Acad Sci 2011;108:10980-5.

23. Singh S, Sharma A, Robertson GP. Realizing the Clinical Potential of Cancer Nanotechnology by Minimizing Toxicologic and Targeted Delivery Concerns. Cancer Res 2012;72:5563-8.

24. Onoue S, Yamada A, Cha HK. Nanodrugs: pharmacokinetics and safety. Int J Nanomedicine 2014;9:1025-37.

25. Gmeiner WH, Ghosh S. Nanotechnology for cancer treatment Nanotechnol Rev 2014;3:111-22.

26. Martins S, Sarmento B, Ferreira DC, Souto EB. Lipid- based collodial carriers for peptide and protein delivery- liposomes versus lipid nanoparticles. Int J Nanomedicine 2007;2:595-607.

27. Shahbazi MA, Santos HA. Improving oral absorption via drug-loaded nanocarriers: absorption mechanisms, intestinal models and rational fabrication. Curr Drug Metab 2013;14:28-56.

28. Thomas N, Holm R, Mullertz A, Rades T. In vitro and in vivo performanceof novel supersaturated self-nanoemulsifying drug delivery systems (super-SNEDDS). J Control Release 2012;160:25-32.

29. Shahbazi MA, Santos HA. Improving oral absorption via drug-loaded nanocarriers: absoption mechanism, intestinal models and rational fabrication. Cur Drug Met 2013;14:28-56.

30. Onoue S, Sato H, Ogawa K, Kojo Y, Aoki Y, Kawabata Y,Wada K,Mizumoto T, Yamada S. Inhalable dry-emulsion formulation of cyclosporin A eith improved anti-inflammatory effects in experimental asthma/COPD-model rats. Eur J Biopharm 2012;80:54-60.

31. Devalapally $H$, Chakilam A, Amji MM. Role of nanotechnology in pharmaceutical product development. J Pharm Sci 2007;96:2547-65.

32. Honda A, Asai T, Oku N, ArakiY, Tanaka M,Ebihara N. Liposomes and nanotechnology in drug development: focus on ocular targets. Int J Nanomedicine 2013;8:495-503.

33. Zhang L, Gu FX, Chan JM, Wang AZ, Lang RS, Farokhzad OC. Nanoparticles in medicine:therapeutic applications and developments. Clin Pharmacol Ther 2008;83:761-9.
34. Barenholz Y. Doxil ${ }^{\circledR}$ - The first FDA-approved nano-drug: Lessons learned. J Control Release 2012;160:117-34.

35. Batist G, Ramakrishnan G, Rao CS, Chandrasekharan A, Gutheil J, Guthrie T, Shah P, Khojasteh A, Nair MK, Hoelzer K, Tkaczuk K, Park YC, Lee LW. Reduced cardiotoxicity and preserved antitumor efficacy of liposome-encapsulated doxorubicin and cyclophosphamide compared with conventional doxorubicin and cyclophosphamide in a randomized, multicenter trial of metastatic breast cancer. J Clin Oncol 2001;19:1444-54.

36. O'Brien ME, Wigler N, Inbar M, Rosso R, Grischke E, Santoro A, Catane R, Kieback DG, Tomczak P, Ackland SP, Orlandi F, Mellars L, Alland L, Tendler C; CAELYX Breast Cancer Study Group. Reduced cardiotoxicity and comparable efficacy in a phase III trial of pegylated liposomal doxorubicin $\mathrm{HCl}$ (CAELYX/Doxil) versus conventional doxorubicin for first-line treatment of metastatic breast cancer. Ann Oncol 2004; 15:440-9.

37. Jehn CF, Hemmati $P$, Lehenbauer-Dehm S, Kümmel S, Flath B, Schmid P. Biweekly Pegylated Liposomal Doxorubicin (Caelyx) in Heavily Pretreated Metastatic Breast Cancer: A Phase 2 Study. Clin Breast Cancer 2016;16:514-9.

38. Minotti G, Menna P, Salvatorelli E, Cairo G, Gianni L Anthracyclines: molecular advances and pharmacologic developments in antitumor activity and cardiotoxicity. Pharmacol Rev 2004;56:185-229.

39. Glantz MJ1, LaFollette S, Jaeckle KA, Shapiro W, Swinnen L, Rozental JR, Phuphanich S, Rogers LR, Gutheil JC, Batchelor T, Lyter D, Chamberlain M, Maria BL, Schiffer C, Bashir R, Thomas D, Cowens W, Howell SB. Randomized trial of a slow-release versus a standard formulation of cytarabine for the intrathecal treatment of lymphomatous meningitis. J Clin Oncol 1999;17:3110-6.

40. http://www.ema.europa.eu/ema/index.jsp?curl=pages/ medicines/human/ medicines/004125/human med_002022. jsp\&mid=WC0b01ac058001d124. [cited 2018 Jul 6]

41. Onoue S, Yamada S, Chan HK. Nanodrugs: pharmacokinetics and safety. Int J Nanomedicine 2014;9:1025-37.

42. Li QW, Cai TG, Huang YH, Xia X, Cole SPC, Cai Y. A Review of the structure, preparation, and application of NLCs, PNPs, and PLNs. Nanomaterials 2017;7(6):pii:E122. doi: 10.3390/nano7060122

43. Wong HL, Bendayan R, Rauth AM, Li Y, Wu XY. Chemotherapy with anticancer drugs encapsulated in solid lipid nanoparticles. Adv Drug Deliv Rev 2007;59:491-504.

44. Din FU, Aman W, Ullah I, Qureshi OS, Mustapha O, Shafique S, Zeb A. Effective use of nanocarriers as drug delivery systems for the treatment of selected tumors. Int J Nanomedicine 2017;12:7291-309.

45. Ho BN, Pfeffer CM, Singh ATK. Update on Nanotechnology-based Drug Delivery Systems in Cancer Treatment. Anticancer Res 2017;37:5975-81

46. Ji Z, Lin G, Lu Q, Meng L, Shen X, Dong L, Fu C, Zhang X. Targeted therapy of SMMC-7721 liver cancer in vitro and in vivo with carbon nanotubes based drug delivery system. J Colloid Interface Sci 2012;365:143-9.

47. Harrington KJ, Mohammadtaghi S, Uster PS, Glass D, Peters AM, Vile RG, Stewart JS. Effective targeting of solid tumors in patients with locally advanced cancers by radiolabeled pegylated liposomes. Clin Cancer Res 2001;7:243-54.

48. Mora-Huertas CE, Fessi $\mathrm{H}$, Elaissari A. Polymer-based nanocapsules for drug delivery. Int J Pharm 2010;385:113-42.

49. Wilczewska AZ, Niemirowicz K, Markiewicz KH, Car H. Nanoparticles as drug delivery systems. Pharmacol Rep 2012;64:1020-37.

50. https://www.cancer.gov/about-cancer/treatment/drugs/fdananoparticle-paclitaxel [cited 2018 Jul 6]

51. Dostalova S, Vasickova K, Hynek D, Krizkova S1, Richtera L, Vaculovicova M, Eckschlager T, Stiborova M, Heger Z, Adam V. Apoferritin as an ubiquitous nanocarrier with excellent shelf life. Int J Nanomedicine 2017;12:2265-78.

52. Dostalova S, Polanska H, Svobodova M, Balvan J, Krystofova O, Haddad Y, Krizkova S, Masarik M, Eckschlager T, Stiborova M, Heger Z, Adam V.Prostate-Specific Membrane Antigen-Targeted Site-Directed Antibody-Conjugated Apoferritin Nanovehicle Favorably Influences In Vivo Side Effects of Doxorubicin. Sci Rep 2018;8:8867.

53. Trotta F, Dianzani C,Caldera F, Mognetti B, Cavalli R. The application of nanosponges to cancer drug delivery. Expert Opin Drug Deliv 2014;11:931-41.

54. Mognetti B, Barberis A, Marino S, Berta G, Francia SD, Trotta F. In vitro enhancement of anticancer activity of paclitaxel by a cremophor 
free cyclodextrin based nanosponge formulation. J Incl Phenom Macrocycl Chem 2012;74:201-10.

55. Kim BYS, Rutka JT, Chan WCW. Nanomedicine NEJM 2010;363:243443.

56. Lee CC, Gillies ER, Fox ME, Guillaudeu SJ, Frechet JMJ, Dy EE, Szoka FC. A single dose of doxorubicin-functionalized bow-tie dendrimer cures mice bearing C-26 colon carcinomas. Proc Natl Acad Sci U S A 2006;103:16649-54.

57. Drbohlavova J, Chomoucka J, Adam V, Ryvolova M, Eckschlager T, Hubalek J, Kizek R. Nanocarriers for anticancer drugs--new trends in nanomedicine. Curr Drug Metab 2013;14:547-64.

58. Hanafy NAN, El-Kemary M, Leporatti S. Micelles Structure Development as a Strategy to Improve Smart Cancer Therapy. Cancers (Basel) 2018;10:pii:E238.

59. Kim TY, Kim DW, Chung JY, Shin SG, Kim SC, Heo DS, Kim NK, Bang YJ. Phase I and pharmacokinetic study of Genexol-PM, a cremophorfree, polymeric micelle-formulated paclitaxel, in patients with advanced malignancies. Clin. Cancer Res 2004;10:3708-16.

60. Aberoumandi SM, Mohammadhosseini M, Abasi E, Saghati S, Nikzamir N, Akbarzadeh A, Panahi Y, Davaran S. An update on application of nanostructured drug delivery systems in cancer therapy: a review. Artif Cell Nanomed Biotechnol 2017;45:1058-68.

61. Hrib J, Sirc J, Hobzova R, Hampejsova Z, Bosakova Z, Munzarova M, Michalek J. Nanofibers for drug delivery - incorporation and release of model molecules, influence of molecular weight and polymer structure. Beilstein J Nanotechnol 2015;6:1939-45.

62. Lu T, Jing $X$, Song $X$, Wang $X$. Doxorubicin-Loaded Ultrafine PEGPLA Fiber Mats Against Hepatocarcinoma. J Appl Polym Sci 2012;123:209-17.

63. Xu X, Chen X, Wang Z, Jing X. Ultrafine PEG-PLA fibers loaded with both paclitaxel and doxorubicin hydrochloride and their in vitro cytotoxicity. Eur J Pharm Biopharm 2009;72:18-25.

64. Wei J, Hu J, Li M, Chen Y, Chen Y. Multiple drug-loaded electrospun PLGA/gelatin composite nanofibers encapsulated with mesoporous $\mathrm{ZnO}$ nanospheres for potential postsurgical cancer treatment. RSC Advances 2014;4:28011-19.

65. Gharpure KM, Wu SY, Li C, Lopez-Berestein G, Sood AK. Nanotechnology: Future of Oncotherapy. Clin Cancer Res 2015;21:3121-30.
66. Tormo M, Tari AM, McDonnell TJ, Cabanillas F, Garcia-Conde J, LopezBerestein G. Apoptotic induction in transformed follicular lymphoma cells by Bcl-2 downregulation. Leuk Lymphoma 1998;30:367-79.

67. Plch J, Venclikova K, Janouskova O, Hrabeta J, Eckschlager T, Kopeckova K, Hampejsova Z, Bosakova Z, Sirc J, Hobzova R. Paclitaxel-Loaded Polylactide/Polyethylene Glycol Fibers with Long-Term Antitumor Activity as a Potential Drug Carrier for Local Chemotherapy. Macromol Biosci 2018;18(6):e1800011

68. Shi J, Kantoff PW, Wooster R, Farokhzad OC. Cancer nanomedicine: progress, challenges and opportunities. Nat Rev Cancer 2016;17:2037.

69. Kaplan JA, Liu R, Freedman JD, Padera R, Schwartz J, Colson YL, Grinstaff MW. Prevention of lung cancer recurrence using cisplatin-loaded superhydrophobic nanofiber meshes. Biomaterials 2016;76:273-81.

70. Ding QX, Li Z, Yang Y, Guo G, Luo F, Chen ZQ, Yang Y, Qian ZY, Shi S. Preparation and therapeutic application of docetaxel-loaded poly $(D, L-l a c t i d e)$ nanofibers in preventing breast cancer recurrence. Drug Deliv 2016;23:2677-85.

71. Ma Y, Wang X, Zong S, Zhang Z, Xie Z, Huang Y, Yue Y, Liu S, Jing X. Local, combination chemotherapy in prevention of cervical cancer recurrence after surgery by using nanofibers co-loaded with cisplatin and curcumin. RSC Advances 2015;5:106325-32.

72. Pillai G. Nanomedicines for cancer therapy: an update of FDA approved and those under various stages of development. SOJ Pharm Pharm Sci 2014;1:13-25.

73. Cerna T, Stiborova M, Adam V, Kizek R, Eckschlager T. Nanocarrier drugs in the treatment of brain tumors J Cancer Metastasis Treat 2016;2:407-16.

74. Dawidczyk CM, Russell LM, Searson PC. Nanomedicines for cancer therapy: state-of-the-art and limitations to pre-clinical studies that hinder future developments, Front Chem 2014;2:69.

75. Kopel P, Blazkova I, Vaculovicova M, Adam V, Eckschlager T, Stiborova M, Kizek R. Characterization of Carbon Nanotubes for Doxorubicin Encapsulation. In: CRC Concise Encyclopedia of Nanotechnology, pp. 186-195. 